\title{
PET radioligand binding to translocator protein (TSPO) is increased in unmedicated depressed subjects
}

Erica M. Richards ${ }^{1 *}$, Paolo Zanotti-Fregonara², Masahiro Fujita', Laura Newman', Cristan Farmer', Elizabeth D. Ballard', Rodrigo Machado-Vieira ${ }^{1,3}$, Peixiong Yuan ${ }^{1}$, Mark J. Niciu', Chul Hyoung Lyoo", Ioline D. Henter ${ }^{1}$, Giacomo Salvadore ${ }^{5}$, Wayne C. Drevets ${ }^{5}$, Hartmuth Kolb ${ }^{5}$, Robert B. Innis ${ }^{1}$ and Carlos A. Zarate $\mathrm{Jr}^{1}$

\begin{abstract}
Background: Inflammation is associated with major depressive disorder (MDD). Translocator protein $18 \mathrm{kDa}$ (TSPO), a putative biomarker of neuroinflammation, is quantified using positron emission tomography (PET) and ${ }^{11} \mathrm{C}-\mathrm{PBR} 28$, a TSPO tracer. We sought to (1) investigate TSPO binding in MDD subjects currently experiencing a major depressive episode, (2) investigate the effects of antidepressants on TSPO binding, and (3) determine the relationship of peripheral and central inflammatory markers to cerebral TSPO binding. Twenty-eight depressed MDD subjects (unmedicated $(n=12)$ or medicated $(n=16))$ and 20 healthy controls $(\mathrm{HC})$ underwent PET imaging using ${ }^{11} \mathrm{C}$-PBR28. Total distribution volume $\left(V_{T}\right.$, proportional to Bmax/Kd) was measured and corrected with the free fraction in plasma $(f p)$. The subgenual prefrontal cortex (sgPFC) and anterior cingulate cortex (ACC) were the primary regions of interest. Peripheral blood samples and cerebrospinal fluid were analyzed to investigate the relationship between TSPO binding and peripheral and central inflammatory markers, including interleukins and neurotrophic factors previously linked to depression.
\end{abstract}

Results: TSPO binding was higher in MDD versus HC in the sgPFC (Cohen's $d=0.64, p=.038,95 \% \mathrm{Cl} 0.04-1.24$ ) and ACC $(d=0.60, p=.049,95 \% \mathrm{Cl} 0.001-1.21)$, though these comparisons missed the corrected threshold for statistical significance $(a=.025)$. Exploratory analyses demonstrated that unmedicated MDD subjects had the highest level of TSPO binding, followed by medicated MDD subjects, who did not differ from HC. TSPO binding correlated with interleukin-5 in cerebrospinal fluid but with no other central inflammatory markers.

Conclusions: This study found a trend towards increased TSPO binding in the brains of MDD subjects, and post hoc analysis extended these findings by demonstrating that this abnormality is significant in unmedicated (but not medicated) MDD subjects.

Keywords: Inflammation, Major depressive disorder, Biomarkers, Peripheral benzodiazepine receptor, Positron emission tomography

\footnotetext{
* Correspondence: ericha25@jhmi.edu

${ }^{1}$ Intramural Research Program, National Institute of Mental Health, National

Institutes of Health, Building 10, CRC Room 6-5340, 10 Center Drive,

Bethesda, MD 20892, USA

Full list of author information is available at the end of the article
} 


\section{Background}

Inflammation is associated with major depressive disorder (MDD), both as a putative causal factor and as a biomarker of disease state and/or response to antidepressant treatment [1, 2]. Medical illnesses associated with peripheral inflammation, such as rheumatoid arthritis, systemic lupus erythematosus, and multiple sclerosis, have higher rates of comorbid MDD compared to the general population [3, 4]. Conversely, some pro-inflammatory markers are normalized in individuals with MDD treated with antidepressant medications or electroconvulsive therapy [58]. While most studies have linked MDD to increased peripheral inflammation, central inflammation-typically measured by evaluating inflammatory biomarkers in cerebrospinal fluid (CSF) - has also been associated with depression $[9,10]$, albeit less consistently.

Tumor necrosis factor-alpha (TNF-a), brain-derived neurotrophic factor (BDNF), C-reactive protein (CRP), and various interleukins (ILs) -including IL-5, IL-6, IL-8, and IL-10-have been implicated in the pathophysiology of MDD [5, 8, 11-15]. In some instances, however, the direction of differences relative to healthy controls have differed between peripheral and central levels of the same cytokine, making it difficult to extrapolate peripheral abnormalities to the brain $[10,16]$.

While differences in inflammatory markers in the blood and CSF are well-documented, it remains unclear whether inflammatory changes manifest in the brains of MDD subjects. Translocator protein $18 \mathrm{kDa}$ (TSPO) is a mitochondrial protein that transports cholesterol to an enzyme that synthesizes pregnenolone, which is a precursor for steroids and neurosteroids [17]. Because TSPO is highly expressed in immune and glial cells within the brain-particularly activated microglia and reactive astrocytes-it is a potential biomarker of neuroinflammation and, furthermore, can be accurately quantified through PET [18]. A 2013 study found no differences in TSPO binding between healthy controls and MDD subjects [19]; however, a recent positron emission tomography (PET) study by Setiawan and colleagues found that, compared to healthy controls, subjects with MDD had increased TSPO density [20]. Using PET imaging and ${ }^{18}$ F-FEPPA, a radioligand for TSPO, the authors found a positive correlation between TSPO binding and severity of depression but observed no correlation between TSPO binding and several peripheral biomarkers of inflammation [20]. Thus, while neuroinflammation and MDD appeared to be linked in some studies, it remains unknown whether these findings are replicable, whether they may be influenced by antidepressant treatment, and whether peripheral markers of inflammation, such as TNF-alpha and other cytokines, correlate with TSPO density.

The primary aim of this study was to investigate TSPO binding in MDD subjects. The secondary aims were to determine if antidepressant use affects TSPO binding and whether TSPO binding is associated with peripheral and/or central biomarkers of inflammation. We conducted PET scans with medicated and unmedicated MDD subjects and healthy controls using ${ }^{11} \mathrm{C}-\mathrm{PBR} 28$ - a TSPO tracer and a close chemical analog of ${ }^{18} \mathrm{~F}-\mathrm{FEPPA}$ - as an indirect measure of neuroinflammation [21]. Both ${ }^{11} \mathrm{C}$-PBR28 and ${ }^{18}$ F-FEPPA are second-generation radioligands and, because TSPO is increased not only in activated microglia but also in reactive astrocytes, both ligands are considered able to indirectly measure neuroinflammation. We also sampled peripheral blood and cerebrospinal fluid to investigate the relationship between TSPO and inflammatory markers.

\section{Methods}

\section{Participants}

Participants included 28 subjects (10 women and 18 men, aged 18 or older) diagnosed with MDD, currently experiencing a moderate-to-severe major depressive episode ( $n=12$ unmedicated and $n=16$ medicated, based on current state at intake) and 20 healthy controls with no current or prior mental health history (10 men and 10 women, aged 18 or older) (Table 1 ). Unmedicated subjects were medication-free for at least 2 weeks prior to the PET scan. All subjects were screened at the Clinical Research Center of the National Institute of Mental Health (NIMH) in Bethesda, Maryland, between January 2014 and March 2016 (ClinicalTrials.gov identifier: NCT01851356). Participants were diagnosed with MDD via an interview with a licensed, independent psychiatrist and confirmed with the Structured Clinical Interview for Axis I DSM-IV Disorders-Patient Version [22]. All subjects completed the Montgomery-Asberg Depression Rating Scale (MADRS) within 1 week prior to the PET scan; inclusion criteria required a score $\geq 20$. Exclusion criteria were evidence of risk of imminent suicide, bipolar disorder, substance abuse within 6 months, or any comorbid illness likely to affect the subject's inflammatory status. The additional exclusion criterion for healthy controls was any history of an Axis I diagnosis. All subjects were screened for the rs6971 polymorphism on the TSPO gene using in vitro TSPO phenotypic binding assays from leukocytes; six low-affinity binders (three MDD, three controls; rate did not differ between groups) were excluded from the study because they do not bind appreciable amounts of TSPO ligands [23].

This study was approved by the Combined Neuroscience Institutional Review Board of the National Institutes of Health. All participants gave written informed consent before entry into the study.

\footnotetext{
${ }^{11}$ C-PBR28 PET imaging

${ }^{11} \mathrm{C}$-PBR28 PET scans were performed on a GE Advance Tomograph (GE Medical Systems; Waukesha, WI). In
} 
Table 1 Demographic information

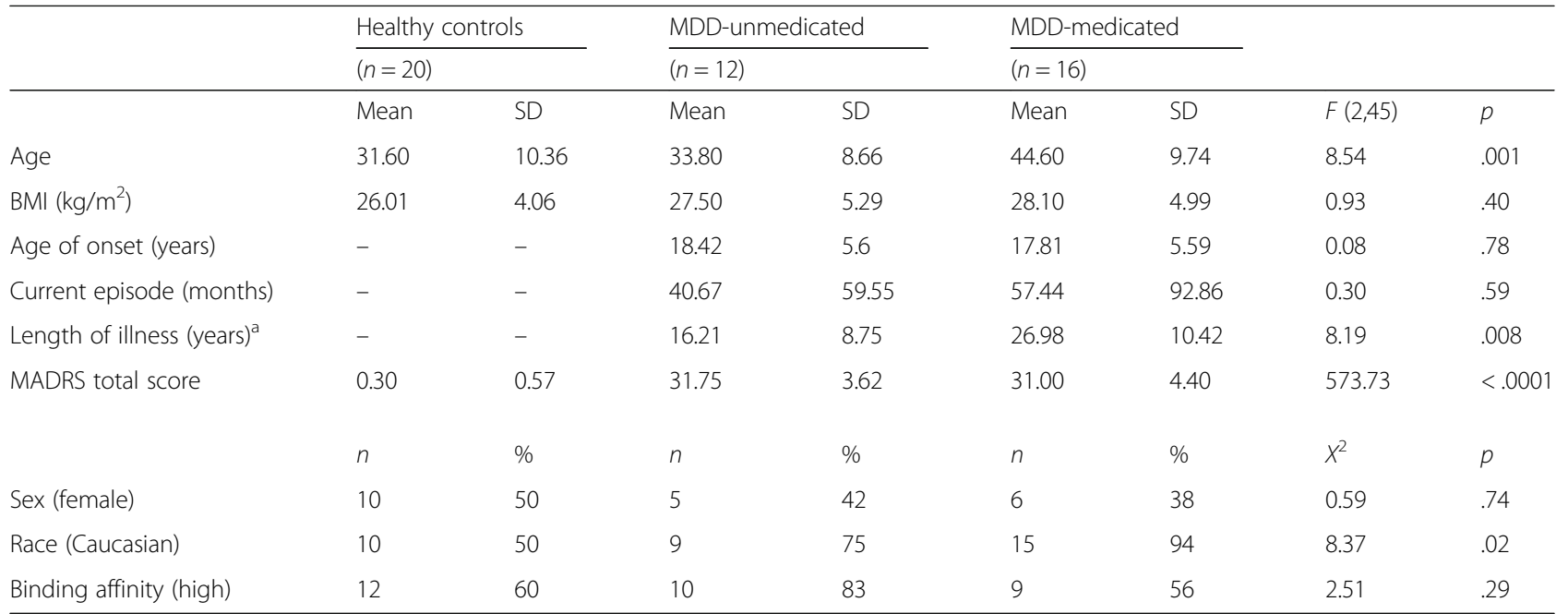

MDD major depressive disorder, BMI body mass index, MADRS Montgomery-Asberg Depression Rating Scale

${ }^{a}$ Missing data for $n=1$ in MDD-medicated

two subjects, the images were instead acquired with a high-resolution research tomograph (Siemens Medical Solutions; Malvern, PA); images for these two subjects were reconstructed using the same resolution and parameters used for the GE scans. After a transmission scan for attenuation correction, ${ }^{11} \mathrm{C}-\mathrm{PBR} 28$ was injected in bolus (healthy volunteers, $701 \pm 19 \mathrm{MBq}$; medicated MDD subjects, $694 \pm 10 \mathrm{MBq}$; unmedicated MDD subjects, $700 \pm 12 \mathrm{MBq}$ ), and the images were acquired in 3D for $90 \mathrm{~min}$. Arterial blood samples were drawn manually at 15 -s intervals for the first $2.5 \mathrm{~min}$, then at 3 , $4,6,8,10,15,20,30,40,50,60,75$, and 90 min. Radioactivity in whole blood and plasma was measured by a gamma counter, and the parent concentration was obtained by high-performance liquid chromatography [24]. The free fraction of ${ }^{11} \mathrm{C}$-PBR28 in plasma $\left(f_{\mathrm{P}}\right)$ was measured by ultrafiltration and normalized using a standard derived from pooled donor plasma to correct for day-to-day intra-assay variability [25] and yielded a value of $2.85 \pm 0.76 \%, 2.98 \pm 0.76 \%$, and $2.49 \pm 0.26 \%$, respectively, for healthy volunteers, medicated, and unmedicated subjects with depression. $f_{\mathrm{P}}$ was not available for one healthy control subject, so the average group value from the other healthy controls was used.

All participants also underwent brain magnetic resonance imaging (MRI) within 1 year prior to the PET scan. T1-weighted MR images were acquired using a 3T Philips Achieva scanner (Bothell, WA) with turbo field echo sequence (repetition time $=8.1 \mathrm{~ms}$, echo time $=3.7 \mathrm{~ms}$, flip angle $=8$, matrix $=181 \times 256 \times 256$, voxel size $=1 \times$ $0.983 \times 0.983 \mathrm{~mm}$ ).

PET brain time-activity curves were obtained with the Pneuro module of Pmod 3.8 (Zurich, Switzerland). Image pre-processing-such as coregistration between
PET and MR, segmentation, and atlas normalizationwas performed with the Pneuro pipeline. The regions of interest were automatically defined using the Hammers' probabilistic brain atlas [26].

\section{Selection of brain regions}

In order to reduce the likelihood of type II errors, we selected two regions of interest a priori: the anterior cingulate cortex (ACC) and the subgenual prefrontal cortex (sgPFC). These regions were selected because they have been shown to differ in individuals with MDD using multiple imaging modalities [27-30] and often normalize following antidepressant treatment [8]. Increased microglial quinolinic acid immunoreactivity in specific subregions of the ACC further underscores the involvement of these regions and supports the role of the immune system in the pathophysiology of MDD [31]. Seventy-five brain regions from both hemispheres-including the insula, putamen, thalamus, hippocampus, and cerebellum-were included in a secondary analysis to determine if changes in TSPO binding were more region-specific or globally distributed in the brain.

ACC and sgPFC values were obtained by averaging the left and right sides of each region. The sgPFC region was obtained by merging the subgenual, subcallosal, and presubgenual areas of the Hammers' atlas.

\section{${ }^{11}$ C-PBR28 PET imaging data analysis}

At the regional level, total distribution volume $\left(V_{\mathrm{T}}\right)$ was calculated using a two-tissue compartmental model and a metabolite-corrected arterial input function fitted to a tri-exponential function. Brain data were weighted frame-wise by assuming that the inverse square root of noise-equivalent counts was proportional to the 
normalized standard deviation of each frame. The arterial whole blood curve was used to correct for activity in the vascular component, assuming that the blood volume is $5 \%$ of the total brain volume. The delay between the arrival of the radioligand to the brain and to the radial artery was estimated by fitting the whole brain curve. $V_{\mathrm{T}}$ values were then corrected for $f_{\mathrm{P}}$ to obtain the final outcome parameter $V_{\mathrm{T}} / f_{\mathrm{p}}$. Uncorrected $V_{\mathrm{T}}$ values were also compared to test the robustness of these findings. Genotype (mixed-affinity vs high-affinity) was added as a covariate to all models.

At the voxel level, we calculated first the parametric images with the Logan plot and divided each image by the individual free fraction. The $V_{\mathrm{T}} / f_{\mathrm{P}}$ images of the three groups were then normalized and smoothed with an 8-mm Gaussian filter. Using SPM12 (Wellcome Trust, London, UK), an ANCOVA model was used for the comparison between the groups with the diagnosis and the genotype status as fixed factors and age and BMI as covariates. We first created significance map with cutoff $p<0.005$ uncorrected for multiple comparisons and then applied cluster-wise correction for multiple comparisons with cluster-level cutoff $p<0.05$.

\section{Peripheral and central biomarker analysis}

All subjects provided peripheral blood samples, which were collected using the vacutainer system within $2 \mathrm{~h}$ before the PET scan. Blood samples were centrifuged at $3000 \mathrm{rpm}$ at $4{ }^{\circ} \mathrm{C}$ for $10 \mathrm{~min}$ and stored at $-80^{\circ} \mathrm{C}$. For those who consented ( $n=6$ healthy controls, $n=4$ unmedicated MDD, $n=10$ medicated MDD), up to $15 \mathrm{~mL}$ CSF was collected within 1 week following the PET scan and immediately placed in liquid nitrogen and stored at $-80{ }^{\circ} \mathrm{C}$.

The following peripheral and central markers of inflammation were examined as correlates of TSPO binding (see Additional file 1 for specific methods): vascular endothelial growth factor (VEGF), IL-6, IL-8, amyloid $\mathrm{A} 1$, adiponectin, BDNF (plasma only, not detected in CSF), TNF-alpha (plasma only), CRP (plasma only), IL-2 (CSF only, not detected in plasma), IL-5 (CSF only), and interferon-gamma (CSF only).

\section{Statistical analysis}

The general linear model was used to evaluate the primary hypothesis, which was that TSPO binding is elevated among patients with MDD relative to $\mathrm{HC}$, and the secondary hypothesis, which was that antidepressant use among MDD patients, would be associated with normalized TSPO binding. Genotype was entered as a covariate; no other covariates were specified a priori (see Additional file 1 for additional information). Partial Pearson correlations were used to evaluate the relationship between TSPO binding and inflammatory markers; body mass index (BMI) was added as a covariate in these analyses. Alpha was set to .025 per region (ACC and sgPFC) for the primary aim, and alpha for the secondary and exploratory aims was unadjusted (.05). Cohen's $d$ effect sizes (with 95\% CI) were calculated using least square mean difference estimates and associated degrees of freedom. Data were analyzed using SAS/STAT Version 9.4.

\section{Results}

Demographic information is presented in Table 1. Most $(n=15)$ subjects in the medicated MDD group were on multiple psychoactive medications (median $=2.5$ ) including selective serotonin reuptake inhibitors, serotoninnorepinephrine reuptake inhibitors, serotonin modulators, tricyclic antidepressants, and/or other psychiatric medications (see Additional file 1 for additional information on concomitant medications).

There was a trend towards higher TSPO binding in MDD versus HC (sgPFC: Cohen's $d=0.64, p=.038,95 \%$ CI 0.04-1.24; ACC: $d=0.60, p=.049,95 \%$ CI 0.001-1.21), though these comparisons missed the corrected significance threshold $(\alpha=.025)$ (see Fig. 1, Additional file 1: Table S1). In secondary analyses, TSPO binding was highest in unmedicated MDD participants, followed by medicated MDD participants and HC, which did not differ from one another (see Fig. 2, Additional file 1: Table S2). For both sgPFC and ACC, the differences between unmedicated MDD participants and HC were large (in both regions $p=.005, d=0.89,95 \%$ CI $0.28-1.50$ ) while the differences between unmedicated and medicated MDD participants were moderate (sgPFC: $p=.056, d=0.59,95 \% \mathrm{CI}$ - 0.02-1.20; ACC: $p=.04, d=0.64,95 \%$ CI 0.03-1.25).

This pattern of results was consistent across most regions of interest (see Additional file 1: Tables S3 and S4). Sensitivity analyses using uncorrected $V_{\mathrm{T}}$ yielded consistent results for MDD versus $\mathrm{HC}$, but all between-group comparisons among unmedicated MDD, medicated MDD, and $\mathrm{HC}$ were non-significant (see Additional file 1: Table S5).

Similar to region analysis, the voxel-wise comparison showed higher ${ }^{11} \mathrm{C}$-PBR28 binding across several regions of the brain of unmedicated MDD subjects compared to healthy controls. Areas of significant difference, after correction for multiple comparisons, were spread mostly across the frontal and temporal lobes, bilaterally (Fig. 3). Even for the liberal uncorrected threshold of $p<0.005$, no cluster survived the correction for multiple comparisons when calculating the difference between medicated subjects and healthy controls and the difference between medicated and unmedicated subjects.

We explored whether any particular drug class was associated with decreased binding. Among the total MDD group, the number of drug classes prescribed was not related to TSPO binding $(p=.40)$. No individual drug class 

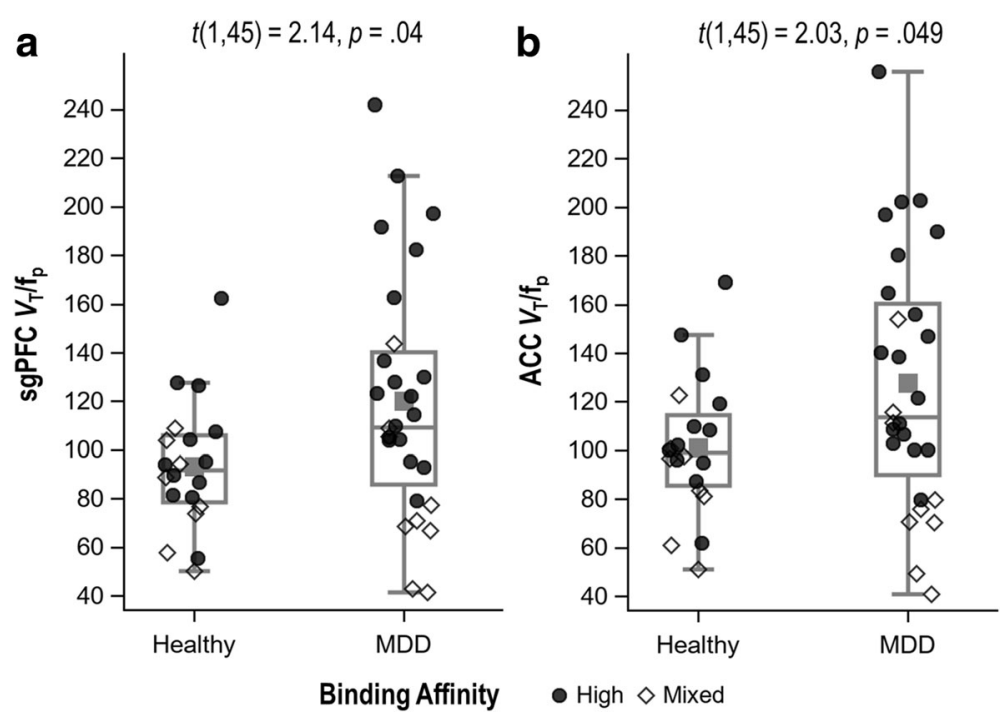

Fig. 1 Translocator protein (TSPO) binding in the subgenual prefrontal cortex (sgPFC) (a) and anterior cingulate cortex (ACC) (b) in individuals with a major depressive disorder (MDD) $(n=28)$ and healthy controls $(n=20)$. Groups are compared using ANCOVA, controlling for genotype

was associated with TSPO binding (all $p>.10$ ). However, because the number of participants prescribed any given drug class was small (see Additional file 1), the power to detect an effect of a given class was low.

No covariates in addition to genotype were specified a priori. However, differences between groups in age and race were observed (see Table 1). Further, a recent study from our laboratory suggested that age is associated with increased TSPO binding in healthy subjects [32], and at least one prior study documented a negative and moderately sized correlation between BMI and TSPO $V_{\mathrm{T}}$ in select brain regions [20]. For these reasons, analyses including age, race, and BMI as additional covariates were performed.

The addition of covariates did not alter the interpretation of the MDD versus $\mathrm{HC}$ analyses (see Additional file 1: Table S1). However, the introduction of additional covariates strengthened the differences between the medicated and unmedicated MDD patients, and between the unmedicated MDD patients and $\mathrm{HC}$, while the medicated MDD patients remained undifferentiated from HC (see Additional file 1: Table S2).

Length of illness in MDD subjects was not related to TSPO binding (sgPFC: $r=.15, p=.46$; ACC: $r=.13$,

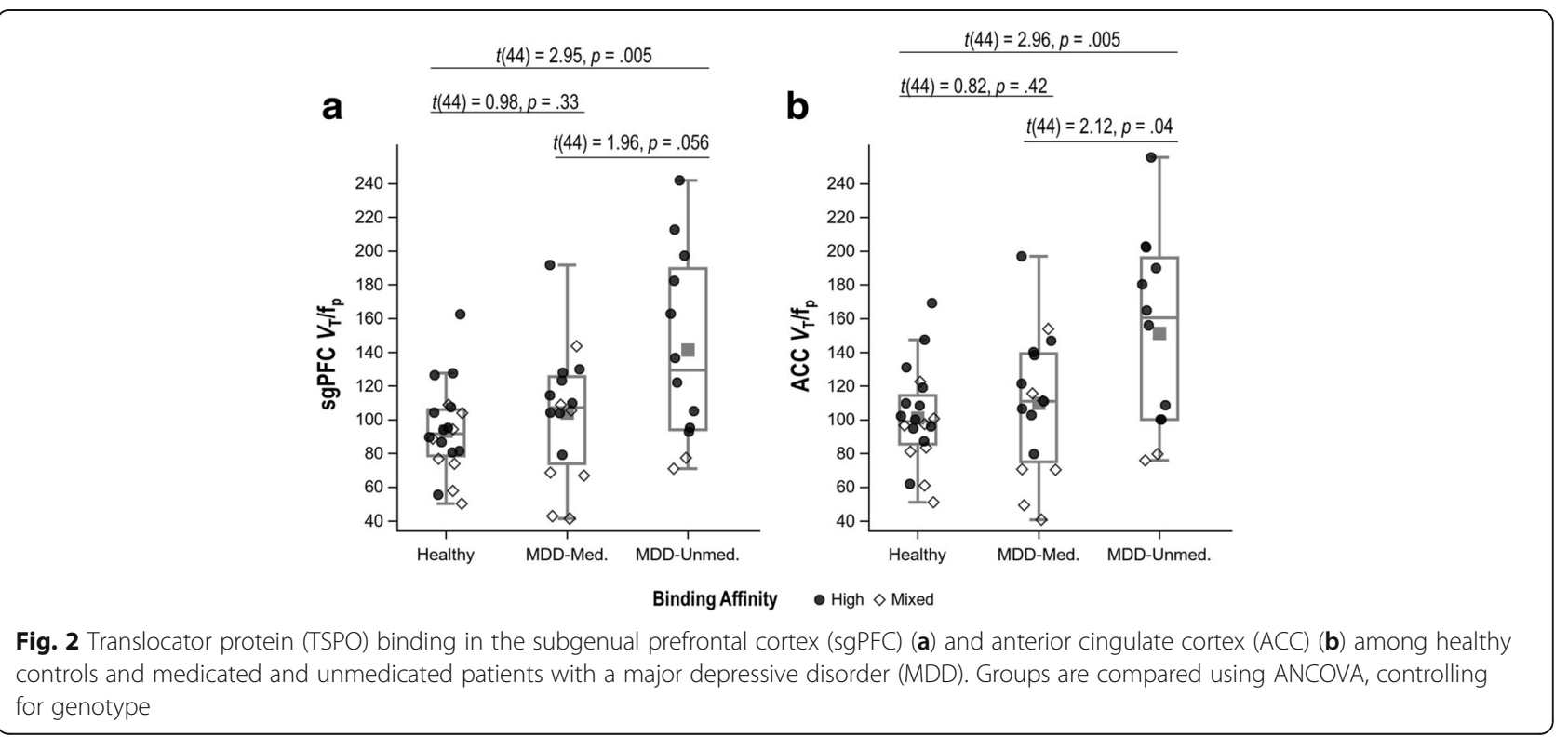




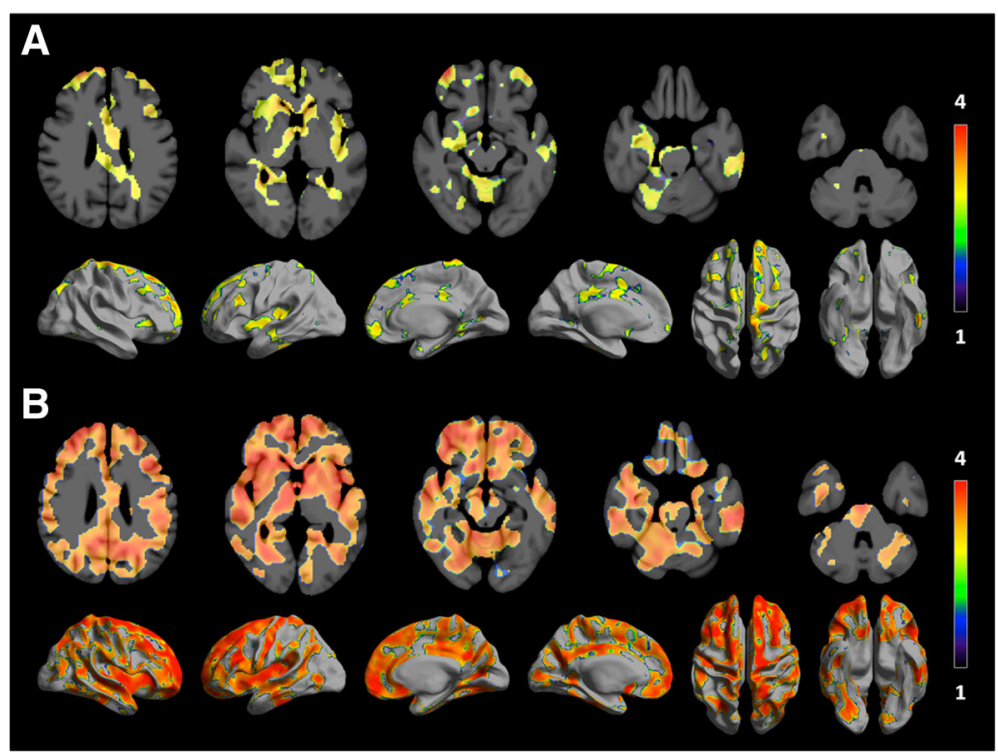

Fig. 3 Statistical significance maps rendered on axial slices and semi-inflated surface showing increase in ${ }^{11} \mathrm{C}$-PBR28 binding in unmedicated depressed patients compared to healthy controls $(\mathbf{a})$. $\mathbf{b}$ The same analysis is restricted to high-affinity binder subjects, done with the more stringent threshold of $p<0.001$. Only clusters with threshold $p<0.05$ for cluster-wise multiple comparisons are displayed. The color bar represents $T$ values. The same comparison using only mixed-affinity binders yielded no significant differences, but the unmedicated group comprised only two mixed-affinity binders

$p=.52)$, and in MDD, TSPO binding did not correlate with MADRS score (sgPFC: $r=-.09, p=.65$; ACC: $r=$ $-.11, p=.56)$. After controlling for BMI, there were no significant between-group (MDD vs HC) differences in inflammatory marker concentrations (data not shown). Only plasma adiponectin and CSF IL-5 were found to be correlated with TSPO binding (Table 2).

Table 2 Partial Pearson correlations, controlling for body mass index, between TSPO binding and central and peripheral markers of inflammation

\begin{tabular}{|c|c|c|c|c|}
\hline & \multicolumn{2}{|c|}{ Plasma $(n=48)$} & \multicolumn{2}{|c|}{$\begin{array}{l}\text { Cerebrospinal fluid } \\
(n=20)\end{array}$} \\
\hline & sgPFC & ACC & sgPFC & ACC \\
\hline Interleukin 2 & - & - & 0.01 & -0.04 \\
\hline Interleukin 5 & - & - & $-0.59^{*}$ & $-0.53^{*}$ \\
\hline Interleukin 6 & -0.16 & -0.15 & -0.25 & -0.26 \\
\hline Interleukin 8 & 0.09 & 0.12 & -0.19 & -0.23 \\
\hline Brain-derived neurotrophic factor & 0.25 & 0.23 & - & - \\
\hline Vascular endothelial growth factor & 0.16 & 0.20 & -0.43 & -0.39 \\
\hline Interferon-gamma & - & - & -0.39 & -0.30 \\
\hline Tumor necrosis factor alpha & -0.01 & 0.02 & - & - \\
\hline C-reactive protein & -0.07 & -0.08 & - & - \\
\hline Amyloid A1 & -0.23 & -0.23 & -0.40 & -0.43 \\
\hline Adiponectin & $0.29^{*}$ & $0.29 *$ & 0.01 & 0.01 \\
\hline
\end{tabular}

sgPFC subgenual prefrontal cortex, ACC anterior cingulate cortex, - data not available ${ }^{*} p \leq .05$

\section{Discussion}

We found increased TSPO binding in the ACC and sgPFC (which were the two regions specified a priori) and throughout the brain of subjects with MDD replicating recent findings [20]. Because increased TSPO binding is an indirect marker of neuroinflammation, these results collectively provide evidence that neuroinflammation is increased in unmedicated MDD subjects and support brain inflammation as a therapeutic target for novel medication interventions. These findings contrast with a prior study that found no difference in TSPO binding between MDD subjects and healthy controls [19]; however, our study differs from that one in that we had a larger sample ( $n=48$ vs 22$)$, with depression that was more severe (average MADRS $=31.5$ vs 19.7) and current (in the previous study, some MDD participants had no current symptoms). We also observed no correlation with MADRS ratings, suggesting that TSPO is not a marker of disease severity in this subject population.

Consistent with the work of Setiawan and colleagues [20], two additional studies demonstrated increased TSPO binding in the brain of patients with MDD [33, 34]. This finding is true when different TSPO radioligands are used $\left({ }^{11} \mathrm{C}\right.$-PBR28 vs ${ }^{18} \mathrm{~F}$-FEPPA $)$. A fourth, smaller $(n=10)$ study documented decreased TSPO binding in MDD, but that study excluded patients with elevated plasma concentrations of C-reactive protein, which may be the subset most likely to show increased 
TSPO in the brain [19]. Thus, this study is the fourth of five to find increased TSPO binding in MDD.

We found that TSPO binding in unmedicated MDD, but not medicated MDD, differed from healthy controls. This suggests that antidepressant treatment itself might normalize TSPO density, even without resolving depressive symptoms. Antidepressant medications have been shown to decrease levels of several peripheral inflammatory markers, including CRP and IL-6 [35, 36]. It is also possible that some SSRIs may decrease microglial activation by inhibiting elevations in intracellular calcium [37]. An experimental study is required to definitively address this question.

We note that although the TSPO density of the medicated MDD did not differ from healthy controls, these subjects were still depressed. This suggests that any anti-inflammatory effects of antidepressant medication, which are thought to act via indirect effects on the inflammatory system, are not sufficient for antidepressant response, though they may still be an important target in a subset of patients. The heterogeneity of MDD highlights the importance of identifying subgroups that may respond to specific interventions. Similar to other studies where subject enrichment helped elucidate specific subgroups who may respond to particular antidepressant interventions (e.g., elevated CRP in studies of inflammation and depression) [38], administering anti-inflammatory treatment interventions to MDD subjects selected for increased TSPO binding may be key to future studies.

Finally, we found that TSPO binding in the sgPFC and ACC correlated significantly and moderately with CSF IL-5 levels; however, no correlation with other peripheral or central markers of inflammation was observed. Overall, no difference in baseline levels of peripheral inflammation was observed between groups, indicating that TSPO binding is not simply a manifestation of changes in underlying baseline peripheral inflammation. These findings suggest that we have yet to identify a specific peripheral blood-based biomarker that may help us understand or predict the inflammatory changes that occur in the brains of subjects experiencing a major depressive episode. Nevertheless, the findings also suggest that additional investigation into IL-5-a key mediator of eosinophil activation-is needed. Some studies have implicated IL-5 in major depressive episodes [5, 39], though others found no significant difference in IL-5 levels between MDD subjects and healthy controls [3]. Our inability to identify biomarkers in CSF or plasma that correlate with TSPO binding likely indicates two key issues, namely, the heterogeneity of the underlying factors contributing to MDD and the limitations of currently available methods for detecting very small changes in blood/CSF biomarker concentrations. Notably, other cytokines and neurotrophic factors have been associated with psychiatric illness and may warrant further investigation. Examples include IL-1 and TGF, which have been implicated in the pathophysiology of multiple psychiatric disorders [40, 41].

Despite these interesting findings, the study has several limitations. First, although increased TSPO has been found in several CNS disorders with localized or widespread neuroinflammation, its cellular localization is not specific activated microglia, which are arguably viewed as the major source of cytokines in the brain. For example, TSPO is also found in astrocytes, including especially high densities in astroglyosis or scars that appear to have no currently active inflammatory processes [42]. In addition, immunohistochemical studies have found that TSPO is associated with vascular endothelium [43]. Although immunohistochemistry lacks the resolution to separate vascular endothelium from the immediately adjacent foot processes of astrocytes, this component of total TSPO binding is certainly not microglial in origin.

\section{Conclusions}

In summary, the relative distribution of TSPO among cells of the brain is unknown, and the increased uptake measured in large regions of the brain with PET and their differing roles, related or unrelated to neuroinflammation, are yet to be clarified. Second, measuring $V_{\mathrm{T}} / f \mathrm{p}$ requires frequent arterial sampling from the subject. While PET studies using TSPO ligands in other disease states (e.g., Alzheimer's disease) have reference regions that may be used instead of arterial sampling [44], the universal increases in TSPO binding observed in unmedicated MDD subjects indicate that no reference region exists in the brain and that arterial sampling will be required to measure differences between patients and controls.

Finally, it should be noted that in the field of mental health, replication studies are rare-largely due to the heterogeneity of patients, relatively small sample sizes available for research studies, and slight differences in experimental design-and the same is true in PET imaging studies. Such research ensures that future planned studies are as informed as possible and allow us, as a field, to move forward in our search to understand the pathophysiology of MDD and develop potential treatments for this devastating disorder. Regarding the present findings, future work may pool the data from this study with others to increase power and generalizability.

In conclusion, TSPO binding is increased in the brains of MDD subjects, and this appears to be diminished with antidepressant treatment. This indirect measurement of neuroinflammation during major depressive episodes suggests that inflammation should continue to be a focus in developing novel therapeutics for the treatment of MDD. 


\section{Additional file}

Additional file 1: Table S1. Results of ANCOVAs comparing TSPO binding $\left(V_{T} / f_{\mathrm{P}}\right)$ in MDD patients to healthy controls (HC). Table S2. Results of ANCOVAs comparing TSPO binding $\left(V_{T} / f_{\mathrm{P}}\right)$ in MDD patients with and without antidepressant medication to healthy controls (HC). Table S3 Results of ANCOVAs comparing TSPO binding $\left(V_{T} / f_{\mathrm{P}}\right)$ in MDD patients to healthy controls $(\mathrm{HC})$ in additional representative regions of interest from the right hemisphere. Table S4. Results of ANCOVAs comparing TSPO binding $\left(V_{T} / f_{\mathrm{P}}\right)$ in healthy controls $(\mathrm{HC})$ to medicated and unmedicated patients with MDD in additional representative regions of interest from the right hemisphere. Table S5. Results of sensitivity analyses using $V_{\mathrm{T}}$. (DOCX $43 \mathrm{~kb}$ )

\section{Acknowledgements}

The authors thank Tessa Walls for the initial administrative support for the project.

\section{Funding}

This study was funded by the Intramural Research Program of the National Institute of Mental Health, National Institutes of Health (projects ZIAMH002852 and ZIAMH002927 under clinical protocol NCT01851356), and by Janssen Pharmaceuticals, Inc.

\section{Availability of data and materials}

Please contact the author for data requests.

\section{Authors' contributions}

ER participated in the design, data collection, and coordination of the study and helped to draft and revise the manuscript. PZF performed the kinetic modeling and image processing and helped to draft and revise the manuscript. MF participated in the design and data collection and helped to draft and revise the manuscript. LN participated in the data collection and coordination of the study. CF performed the statistical analyses and helped to draft and revise the manuscript, EB participated in the data collection and coordination of the study. RMV participated in the design, data collection, and coordination of the study. PY participated in the data collection and coordination of the study. MN participated in the data collection and coordination of the study. CHL participated in the statistical analysis, image processing, and writing of the manuscript. $\mathrm{IH}$ helped summarize the results, helped write the first draft, and critically revised the manuscript. GS conceived the study and participated in the design and coordination of the study. WD participated in the design and coordination of the study. HK participated in the design and coordination of the study. RI conceived the study and participated in its design and coordination. CZ conceived the study and participated in its design and coordination. All authors read and approved the final manuscript.

\section{Ethics approval and consent to participate}

This study was approved by the Combined Neuroscience Institutional Review Board of the National Institutes of Health. All participants gave written informed consent before entry into the study.

\section{Competing interests}

Drs. Salvadore, Drevets, and Kolb are full-time employees and shareholders of Janssen Pharmaceuticals, Inc. Dr. Zarate is listed as a co-inventor on a patent for the use of ketamine in major depression and suicidal ideation; as a co-inventor on a patent for the use of $(2 R, 6 R)$-hydroxynorketamine, (S)dehydronorketamine, and other stereoisomeric dehydro and hydroxylated metabolites of $(R, S)$-ketamine metabolites in the treatment of depression and neuropathic pain; and as a co-inventor on a patent application for the use of $(2 R, 6 R)$-hydroxynorketamine and $(25,65)$-hydroxynorketamine in the treatment of depression, anxiety, anhedonia, suicidal ideation, and post-traumatic stress disorders. He has assigned his patent rights to the US government but will share a percentage of any royalties that may be received by the government. All other authors have no conflict of interest to disclose, financial or otherwise.

\section{Publisher's Note}

Springer Nature remains neutral with regard to jurisdictional claims in published maps and institutional affiliations.

\section{Author details}

Intramural Research Program, National Institute of Mental Health, National Institutes of Health, Building 10, CRC Room 6-5340, 10 Center Drive, Bethesda, MD 20892, USA. ${ }^{2}$ Houston Methodist Research Institute, Weill Cornell Medicine, Houston, Texas, USA. ${ }^{3}$ Department of Psychiatry and Behavioral Sciences, University of Texas Health Science Center, Houston, TX, USA. ${ }^{4}$ Department of Neurology, Gangnam Severance Hospital, Yonsei University College of Medicine, Seoul, South Korea. ${ }^{5}$ Janssen Research and Development, LLC, Titusville, NJ, USA.

Received: 5 March 2018 Accepted: 30 May 2018

Published online: 03 July 2018

\section{References}

1. Raison $\mathrm{CL}$, Miller $\mathrm{AH}$. Is depression an inflammatory disorder? Curr Psychiatry Rep. 2011;13:467-75.

2. Dantzer R, O'Connor JC, Freund GG, Johnson RW, Kelley KW. From inflammation to sickness and depression: when the immune system subjugates the brain. Nat Rev Neurosci. 2008;9:46-56.

3. Kohler CA, Freitas TH, Stubbs B, et al. Peripheral alterations in cytokine and chemokine levels after antidepressant drug treatment for major depressive disorder: systematic review and meta-analysis. Mol Neurobiol. 2017; [Epub ahead of print]

4. Morris G, Berk M, Puri BK. A comparison of neuroimaging abnormalities in multiple sclerosis, major depression and chronic fatigue syndrome (myalgic encephalomyelitis): is there a common cause? Mol Neurobiol. 2017; [Epub ahead of print]

5. Dahl J, Ormstad H, Aass HC, et al. The plasma levels of various cytokines are increased during ongoing depression and are reduced to normal levels after recovery. Psychoneuroendocrinology. 2014;45:77-86.

6. Freire TFV, Rocha NSD, Fleck MPA. The association of electroconvulsive therapy to pharmacological treatment and its influence on cytokines. J Psychiatr Res. 2017;92:205-11.

7. Kim YK, Suh IB, Kim H, et al. The plasma levels of interleukin-12 in schizophrenia, major depression, and bipolar mania: effects of psychotropic drugs. Mol Psychiatry. 2002;7:1107-14

8. Więdłocha M, Marcinowicz P, Krupa R, et al. Effect of antidepressant treatment on peripheral inflammation markers - a meta-analysis. Prog Neuro-Psychopharmacol Biol Psychiatry. 2017; [Epub ahead of print]

9. Hattori K, Ota M, Sasayama D, et al. Increased cerebrospinal fluid fibrinogen in major depressive disorder. Sci Rep. 2015;5:11412.

10. Lindqvist $D$, Janelidze $S$, Hagell $P$, et al. Interleukin-6 is elevated in the cerebrospinal fluid of suicide attempters and related to symptom severity. Biol Psychiatry. 2009;66:287-92

11. Jeenger J, Sharma M, Mathur DM, Amandeep. Associations of number and severity of depressive episodes with C-reactive protein and Interleukin-6. Asian J Psychiatr. 2017;27:71-5.

12. Brenner $\mathrm{P}$, Granqvist M, Konigsson J, et al. Depression and fatigue in multiple sclerosis: Relation to exposure to violence and cerebrospinal fluid immunomarkers. Psychoneuroendocrinology. 2018;89:53-8.

13. Mikova O, Yakimova R, Bosmans E, Kenis G, Maes M. Increased serum tumor necrosis factor alpha concentrations in major depression and multiple sclerosis. Eur Neuropsychopharmacol. 2001;11:203-8.

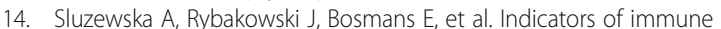
activation in major depression. Psychiatry Res. 1996;64:161-7.

15. Valkanova V, Ebmeier KP, Allan CL. CRP, IL-6 and depression: a systematic review and meta-analysis of longitudinal studies. J Affect Disord. 2013;150: 736-44.

16. Sasayama D, Hattori K, Wakabayashi C, et al. Increased cerebrospinal fluid interleukin-6 levels in patients with schizophrenia and those with major depressive disorder. J Psychiatr Res. 2013;47:401-6.

17. Rupprecht R, Rammes G, Eser D, et al. Translocator protein (18 kD) as target for anxiolytics without benzodiazepine-like side effects. Science. 2009;325: 490-3.

18. Chen MK, Guilarte TR. Translocator protein $18 \mathrm{kDa}$ (TSPO): molecular sensor of brain injury and repair. Pharmacol Ther. 2008;118:1-17.

19. Hannestad J, DellaGioia N, Gallezot JD, et al. The neuroinflammation marker translocator protein is not elevated in individuals with mild-tomoderate depression: a [11C]PBR28 PET study. Brain Behav Immun. 2013:33:131-8. 
20. Setiawan E, Wilson AA, Mizrahi R, et al. Role of translocator protein density, a marker of neuroinflammation, in the brain during major depressive episodes. JAMA Psychiatry. 2015;72:268-75.

21. Fujita M, Imaizumi M, Zoghbi SS, et al. Kinetic analysis in healthy humans of a novel positron emission tomography radioligand to image the peripheral benzodiazepine receptor, a potential biomarker for inflammation. Neurolmage. 2008;40:43-52.

22. First MB, Spitzer RL, Gibbon M, JBW W. Structured Clinical Interview for DSM-IV-TR Axis I Disorders Research Version, patient edition. New York, NY: Biometrics Research, New York State Psychiatric Institute; 2001.

23. Owen DR, Yeo AJ, Gunn RN, et al. An 18-kDa translocator protein (TSPO) polymorphism explains differences in binding affinity of the PET radioligand PBR28. J Cereb Blood Flow Metab. 2012;32:1-5.

24. Zoghbi SS, Shetty HU, Ichise M, et al. PET imaging of the dopamine transporter with 18F-FECNT: a polar radiometabolite confounds brain radioligand measurements. J Nucl Med. 2006;47:520-7.

25. Abi-Dargham A, Gandelman M, Zoghbi SS, et al. Reproducibility of SPECT measurement of benzodiazepine receptors in human brain with iodine-123iomazenil. J Nucl Med. 1995;36:167-75.

26. Hammers A, Allom R, Koepp MJ, et al. Three-dimensional maximum probability atlas of the human brain, with particular reference to the temporal lobe. Hum Brain Mapp. 2003;19:224-47.

27. Philippi CL, Motzkin JC, Pujara MS, Koenigs M. Subclinical depression severity is associated with distinct patterns of functional connectivity for subregions of anterior cingulate cortex. J Psychiatr Res. 2015;71:103-11.

28. Davey CG, Harrison BJ, Yucel M, Allen NB. Regionally specific alterations in functional connectivity of the anterior cingulate cortex in major depressive disorder. Psychol Med. 2012;42:2071-81.

29. Connolly CG, Wu J, Ho TC, et al. Resting-state functional connectivity of subgenual anterior cingulate cortex in depressed adolescents. Biol Psychiatry. 2013;74:898-907.

30. Drevets WC, Price JL, Simpson JR Jr, et al. Subgenual prefrontal cortex abnormalities in mood disorders. Nature. 1997;386:824-7.

31. Steiner J, Walter M, Gos T, et al. Severe depression is associated with increased microglial quinolinic acid in subregions of the anterior cingulate gyrus: evidence for an immune-modulated glutamatergic neurotransmission? J Neuroinflammation. 2011;8:94.

32. Paul S, Gallagher E, Liow JS, et al. Building a database for brain $18 \mathrm{kDa}$ translocator protein (TSPO) imaged using [11C]PBR28 in healthy subjects. J Cereb Blood Flow Metab. 2018. [epub ahead of print]

33. Holmes SE, Hinz R, Conen S, et al. Elevated translocator protein in anterior cingulate in major depression and a role for inflammation in suicidal thinking: a positron emission tomography study. Biol Psychiatry. 2018; 83:61-9.

34. Li L, Wang W, Zhang LM, et al. Overexpression of the $18 \mathrm{kDa}$ translocator protein (TSPO) in the hippocampal dentate gyrus produced anxiolytic and antidepressant-like behavioural effects. Neuropharmacology. 2017;125: 117-28.

35. Abbasi SH, Hosseini F, Modabbernia A, Ashrafi M, Akhondzadeh S. Effect of celecoxib add-on treatment on symptoms and serum IL-6 concentrations in patients with major depressive disorder: randomized double-blind placebocontrolled study. J Affect Disord. 2012;141:308-14.

36. Tynan RJ, Weidenhofer J, Hinwood M, Cairns MJ, Day TA, Walker FR. A comparative examination of the anti-inflammatory effects of SSRI and SNRI antidepressants on LPS stimulated microglia. Brain Behav Immun. 2012;26: 469-79.

37. Horikawa H, Kato TA, Mizoguchi Y, et al. Inhibitory effects of SSRIs on IFN- $\gamma$ induced microglial activation through the regulation of intracellular calcium. Prog Neuro-Psychopharmacol Biol Psychiatry. 2010;34:1306-16.

38. Raison $\mathrm{CL}$, Rutherford RE, Woolwine $\mathrm{BJ}$, et al. A randomized controlled trial of the tumor necrosis factor antagonist infliximab for treatment-resistant depression: the role of baseline inflammatory biomarkers. JAMA Psychiatry. 2013;70:31-41

39. Elomaa AP, Niskanen L, Herzig KH, et al. Elevated levels of serum IL-5 are associated with an increased likelihood of major depressive disorder. BMC Psychiatry. 2012;12:2.

40. Brambilla F, Monti D, Franceschi C. Plasma concentrations of interleukin-1beta, interleukin-6 and tumor necrosis factor-alpha, and of their soluble receptors and receptor antagonist in anorexia nervosa. Psychiatry Res. 2001; 103:107-14.
41. Walss-Bass C, Suchting R, Olvera RL, Williamson DE. Inflammatory markers as predictors of depression and anxiety in adolescents: statistical model building with component-wise gradient boosting. J Affect Disord. 2018;234: 276-81.

42. Johnson EW, de Lanerolle NC, Kim JH, et al. "Central" and "peripheral" benzodiazepine receptors: opposite changes in human epileptogenic tissue. Neurology. 1992;42:811-5.

43. Turkheimer FE, Edison $\mathrm{P}$, Pavese $\mathrm{N}$, et al. Reference and target region modeling of [11C]-(R)-PK11195 brain studies. J Nucl Med. 2007;48:158-67.

44. Lyoo CH, Ikawa M, Liow JS, et al. Cerebellum can serve as a pseudoreference region in Alzheimer disease to detect neuroinflammation measured with PET radioligand binding to translocator protein. J Nucl Med. 2015;56:701-6.

\section{Submit your manuscript to a SpringerOpen ${ }^{\circ}$ journal and benefit from:}

- Convenient online submission

- Rigorous peer review

- Open access: articles freely available online

- High visibility within the field

- Retaining the copyright to your article

Submit your next manuscript at springeropen.com 